\title{
Avaliação das competências de professores de enfermagem para desenvolver programas educativos para adultos
}

\author{
Evaluation of nursing teachers' competencies to develop educational programs for adults
}

Evaluación de las competencias de profesores de enfermería para desarrollar programas educativos para adultos

\author{
Patricia Bover Draganov', Maria Cristina Sanna" \\ 'Universidade Federal de São Paulo, Escola Paulista de Enfermagem, \\ Programa de Pós-Graduação em Enfermagem (Mestranda). São Paulo-SP, Brasil. \\ "Universidade Federal de São Paulo, Escola Paulista de Enfermagem, \\ Programa de Pós-Graduação em Enfermagem. São Paulo-SP, Brasil.
}

\section{Submissão: 25-06-2012 Aprovação: 31-05-2013}

\section{RESUMO}

Avaliar o desempenho que o enfermeiro docente de enfermagem atribui a si, nas competências para desenvolver programas educativos para adultos, foi objetivo desse estudo descritivo, comparativo, transversal e quantitativo, desenvolvido com 226 docentes enfermeiros de cursos de graduação em enfermagem paulistanos. Os dados foram colhidos por escala de Likert, lançados em planilha Excel ${ }^{\circledR}$ e analisados por estatística descritiva e testes não paramétricos de Wilcoxon e Friedman. A população tinha predominantemente entre 46-55 anos (87/38,49\%), eram mulheres (198/87, 61\%) e mestres $(180 / 79,65 \%)$. Os professores consideraram-se distantes das competências que almejavam alcançar. A menor habilidade esteve em construir desenhos de programas para atender às situações de aprendizagem e a maior, em avaliar programas de ensino. O maior desejo esteve em desenhar programas com formatos criativos e o menor desejo para usar conselhos, comitês e força-tarefa. Concluiuse que desenhar programas educativos para adultos ainda é algo pouco explorado e pouco experenciado por esses docentes.

Descritores: Enfermagem; Educação Superior; Docentes de Enfermagem; Modelos Educacionais; Competência Profissional.

\section{ABSTRACT}

To evaluate the performance that nursing teachers assigned to themselves in the skills to develop educational programs for adults, was the aim of this descriptive, comparative, cross-sectional study, performed with 226 teachers of undergraduate nursing courses from Sao Paulo. Data were collected by Likert scale, introduced in Excel ${ }^{\circledR}$ spreadsheet and analyzed using descriptive statistics and nonparametric tests of Wilcoxon and Friedman. The population was predominantly between 46-55 years $(87 / 38,49 \%)$ were women $(198 / 87,61 \%)$ and have a master degree $(180 / 79,65 \%)$. The teachers were far from reaching the competences which they sought. The lower skill was in building the design of programs to meet the learning situations and, the bigger, in evaluating educational programs. The greatest desire was to design programs with creative formats, and the smaller, was to use councils, committees and task force. It was concluded that designing educational programs for adults is still something unexplored and little experienced by these teachers.

Key words: Nursing; Higher Education; Faculty of Nursing; Educational Models; Professional Competence.

\section{RESUMEN}

Estudio descriptivo, comparativo, transversal, envolviendo 226 docentes de cursos de graduación en enfermería, en Sao Paulo-SP, Brasil. Objetivó-se evaluar el desempeño que estos docentes atribuyen a sí mismos, respecto a habilidades para desarrollar programas educativos para adultos. Los datos fueron recogidos mediante una escala tipo Likert, e introducidos en una hoja Excel ${ }^{\circledR}$; se analizaron mediante estadística descriptiva y pruebas no paramétricas de Wilcoxon y Friedman. La población tenía predominantemente entre 46-55 años (87/38, el 49\%), eran mujeres (198/87, 61), con grado de maestría (180/79, el 65\%). Los profesores consideraron que sus habilidades están lejanas al que buscaban alcanzar. La habilidad considerada más baja fue la construcción de diseños de programas para satisfacer situaciones de aprendizaje, y la más grande fue la capacidad para evaluar programas de enseñanza. El mayor deseo era relacionado al diseño de programas con formatos creativos, y el menor fue para usar consejos, comités y grupo de trabajo. Se concluyó que el diseño de programas educativos para los adultos es todavía algo inexplorado y experenciado poco a estos maestros. Palabras clave: Enfermería; Educación Superior; Facultad de Enfermería; Modelos Educativos; Competencia Profesional.

\section{AUTOR CORRESPONDENTEＰatricia Bover Draganov E-mail: patricia.bover@ig.com.br}

Extraído da Dissertação de Mestrado intitulada Avaliação das Competências Andragógicas dos Enfermeiros Docentes de Cursos de Graduação em Enfermagem do Município de São Paulo, apresentada, em 2011, à Universidade Federal de São Paulo,

Programa de Pós-Graduação em Enfermagem. São Paulo-SP, Brasil. 


\section{INTRODUÇÃO}

O processo de educação de adultos sempre esteve articulado às transformações sociais, à evolução do homem, à percepção que este tem sobre si e de seu amadurecimento. A Andragogia é uma teoria que surgiu nesse contexto e esse termo consagrou-se em 1968, quando Malcom Shepherd Knowles passou a utilizá-lo para nomear a teoria e a prática da educação de adultos ${ }^{(1-4)}$.

Nos países latino-americanos, o uso da Andragogia iniciou-se com o projeto da Fundação W. K. Kellogg, denominado "Uma Nova Iniciativa na Formação dos Profissionais de Saúde: União com a Comunidade - UNI", que estimulou o intercâmbio de metodologias de ensino entre países latino-americanos, a partir da década de $1990^{(5)}$, abrangendo cursos de enfermagem. Pesquisas sobre a andragogia na saúde e na enfermagem demonstraram que se trata de um tema impactante e que deverá se manter em destaque no futuro ${ }^{(6-7)}$.

Em 1981, Knowles publicou um instrumento denominado - "Escala de Classificação Autodiagnostica para a Educação de Adultos", com base em três categorias de competências que o professor deveria desenvolver para atuar com adultos: a de facilitador da aprendizagem, de desenvolvedor e a de administrador de programas educativos para adultos ${ }^{(4)}$. No presente texto, será abordada a competência dos professores para desenvolver programas educativos para adultos.

A categoria competências para desenvolver programas educativos para adultos se subdivide em dois domínios - compreender o planejamento do processo e planejar e aplicar programas. Nesse nível de administração, os professores administradores são responsáveis por desenvolver programas, materiais, técnicas, professores (nas competências para a educação de adultos) e pesquisas, em geral, de forma a promover o acréscimo de conhecimento no campo da educação de adultos ${ }^{(8)}$.

Desenhar programas educativos na perspectiva andragógica é uma tarefa que requer preparo e experiência e comporta um processo bastante diferente da pedagogia tradicional que costumeiramente é utilizada na educação de adultos. Além disso, ensinar é um dos processos de trabalho do enfermeiro, e esse processo é sustentado por leis, decretos e diretrizes que recomendam a formação, a educação permanente de enfermeiros e a adaptação constante às mudanças e à vida em sociedade. O professor, nessa perspectiva, deve deter competências inclusive para desenvolver programas educativos para a educação de adultos.

Diante do exposto, questiona-se: o enfermeiro docente de escolas de nível superior paulistanas domina as competências necessárias para desenvolver programas educativos para adultos na perspectiva andragógica?

Vale ressaltar que o índice de crescimento de escolas de formação de enfermeiros no Brasil aumentou consideravelmente desde 1990. Conforme dados do Ministério da Educação $(M E C)^{(9)}$, de 1991 a 2004, em São Paulo, houve crescimento de $263,3 \%$ no número de cursos e, como consequência, o número de docentes aumentou para atender à demanda. Questiona-se se esse aumento súbito foi suficiente para estimular esses docentes a se formarem com as competências necessárias para educar adultos, na graduação em enfermagem.
Acrescenta-se que, de 2004 a 2007, houve crescimento de $39,3 \%$ de estudantes e de $34,8 \%$ de cursos em todo território brasileiro $^{(10)}$, o que também pode ter impulsionado a busca pela formação docente.

A estrutura das escolas também é uma preocupação, uma vez que os dados do MEC, com relação ao Exame Nacional de Desempenho dos Estudantes (Enade), permitiu observar que há problemas na formação de enfermeiros, visto que a média geral da Enfermagem $(31,9 \%)$ foi inferior à média geral da Saúde $(32,3 \%)$, em 2004 ${ }^{(11)}$. O comportamento do Enade ${ }^{(10)}$ de 2007 foi semelhante ao de 2004: o resultado geral mostrou que o rendimento médio geral ficou abaixo do desejado, indicando situação preocupante e necessidade de reflexão sobre a efetividade das práticas educativas. As notas do Enade objetivam refletir o desempenho dos estudantes e o grau de domínio das diversas competências, saberes e conteúdos previstos nas Diretrizes Curriculares Nacionais ${ }^{(11)}$. Notas baixas podem indicar formação profissional de enfermeiros aquém das recomendações do MEC e das competências necessárias para atuar profissionalmente. Mais tarde, essa formação certamente irá se refletir negativamente no desempenho dos processos de trabalho do enfermeiro, sendo que sua formação só pode se dar, com qualidade, em escolas que tenham essa preocupação.

Escolas que promovem boa formação são representadas por pessoas que desejam ensinar (docentes) e outras que desejam aprender (alunos); por esse motivo, olhar para o professor e para as competências que este deve deter, no contexto atual, é tão importante. Bons enfermeiros são formados por docentes competentes, no caso, que detenham, entre outras, as competências para desenvolver programas educativos para a aprendizagem de adultos.

Por fim, ressalta-se que há, na literatura brasileira, poucos estudos que abordem competências de professores para desenvolver programas educativos para a educação de adultos. Na Enfermagem, não há estudos que mensurem as competências de professores enfermeiros que atuam na graduação de enfermagem na perspectiva andragógica, com esse foco. Assim, justifica-se a realização da presente pesquisa.

A presente pesquisa teve como objetivos: 1) Caracterizar a população de enfermeiros docentes de enfermagem que atuavam em cursos presenciais de graduação em enfermagem no município de São Paulo, em 2010; 2) Identificar como o enfermeiro docente de enfermagem avaliou as competências que possui para desenhar programas educativos para adultos e quais considerava ideal ter.

\section{MÉTODO}

Estudo descritivo e comparativo, transversal, com abordagem quantitativa, que foi desenvolvido junto aos cursos de Graduação em Enfermagem, presenciais, autorizados e em funcionamento no município de São Paulo, em 2010. Segundo dados do $\mathrm{MEC}^{(12)}$, nesse ano havia 27 cursos nessa condição, concordando em participar do presente estudo 20 instituições de ensino superior.

A população do estudo compreendeu enfermeiros professores de cursos de graduação presenciais em Enfermagem 
que estavam localizados e funcionando no município de São Paulo; que possuíssem, no mínimo, um curso de graduação presencial em Enfermagem com pelo menos dois anos de funcionamento e estivesse em atividade, e autorizassem, por meio de seus diretores/coordenadores, a abordagem do corpo docente. Os docentes que compuseram a população assinaram o termo de consentimento livre e esclarecido (TCLE); não estavam afastados de suas atividades e exerciam a atividade docente por um período superior a seis meses.

O projeto obedeceu à Resolução 196/96 do Conselho Nacional de Saúde do Ministério da Saúde (MS), de 1996, tendo sido submetido ao Comitê de Ética em Pesquisa com Seres Humanos da Universidade Federal de São Paulo (UNIFESP), sendo aprovado sob o número 1620/10.

O contato com o corpo docente partiu da informação dos coordenadores dos cursos sobre o número total de docentes, somando 653 sujeitos. Foram distribuídos 653 questionários e retornaram $226(34,61 \%)$ instrumentos respondidos e com TCLE assinados.

O instrumento de coleta de dados foi composto de duas partes: dados de caracterização da população e escala de medida tipo Likert, sobre competências para o papel do educador/instrutor de adultos. A escala utilizada foi proposta e testada por Malcom Shepherd Knowles, e a permissão de seu uso é garantida sem limitações ${ }^{(1)}$. Denominado "Escala de Classificação Autodiagnóstica de Competências para o Papel do Educador de Adultos", o instrumento se compõe de três agrupamentos de competências que somam 54 asserções. O agrupamento para desenvolver programas educativos para adultos se compõe dos domínios - I - compreensão do planejamento do processo com quatro habilidades e II - planejamento e aplicação de programas, com cinco habilidades.

A cada descrição de habilidades correspondia uma escala de medida de seis gradações, em que o docente sujeito da pesquisa anotou, utilizando a letra " $\mathrm{A}$ ", o ponto em que avaliava se encontrar atualmente, e a letra " $\mathrm{D}$ ", onde desejava estar. Tratou-se de uma autoavaliação sobre as competências atuais e desejadas do professor, para desenvolver programas educativos.

A coleta de dados se iniciou em junho de 2010, após a obtenção da autorização dos diretores/coordenadores dos cursos e mediante a assinatura do TCLE, pelos docentes, e encerrou-se em fevereiro de 2011.

As informações foram coletadas por meio do instrumento descrito e transcritas para um banco de dados construído com a ferramenta Excel $^{\oplus}$, composto por 119 colunas, com os dados de caracterização da população e resultados da escala. Depois de preenchido o banco, os dados da planilha foram transportados para software para tratamento estatístico denominado Minitab versão 16. No presente texto, apenas os dados de caracterização da população e a parte da escala referente às competências em foco foram relatados. Os demais podem ser aferidos na obra de Draganov ${ }^{(13)}$.
A análise dos dados de caracterização da população do estudo empregou estatística descritiva - frequência simples (número absoluto) e relativa (porcentagem), medidas de tendência central e de variabilidade, ou seja, média, mediana, desvio padrão, valor mínimo e máximo.

Os dados da Escala de Avaliação Autodiagnóstica para o Papel de Educador de Adultos foram tratados em três etapas, com foco no desenvolvimento de programas educativos: 1) Os níveis atual e desejado dos docentes enfermeiros para cada habilidade que compõe um domínio da competência em foco, 2) Os níveis atual e desejado dos docentes enfermeiros para cada um dos domínios da competência em foco e 3) Os níveis atual e desejado dos docentes enfermeiros de todos os domínios de uma vez. Assim, cada linha da escala foi tratada separadamente, utilizando estatística descritiva - soma, proporção (\%), média, desvio padrão, mínimo, mediana e máximo e, após, para a comparação entre os níveis de competência atual ("A") e desejado (" $\mathrm{D}$ "), utilizou-se o teste não paramétrico de Wilcoxon para a primeira e segunda etapa, e Friedman para a terceira. O índice de significância, que determinou se o grau de diferença entre elas era ou não relevante, foi considerado para os valores de $\mathrm{P}<0,05$ ou $5 \%$.

Os dados que resultaram da análise descrita acima foram confrontados com a literatura científica sobre o assunto para discussão dos resultados.

\section{RESULTADOS}

Os professores tinham entre 27 e 67 anos, sendo que 119 $(52,65 \%)$ tinham entre 41 a 55 anos e era, na maioria, 198 $(87,61 \%)$, do sexo feminino. Uma boa parte, $158(69,91 \%)$, trabalhava de 31 a 40 horas semanais e tinha de seis a 10 anos de docência. $176(77,88 \%)$ atuavam em disciplinas teóricas e práticas. $122(53,98 \%)$ se formaram em escola pública, 201 $(88,94 \%)$ fizeram especialização, principalmente em Educação, Administração e Saúde Materno-infantil, 180 (79,65\%) fizeram mestrado, principalmente em Enfermagem em Saúde do Adulto e também em Administração, 77 (34,07\%) professores eram doutores, principalmente em Enfermagem, apenas 3 $(1,33 \%)$ declararam serem livres-docentes e $28(12,39 \%)$ cursaram outra graduação. Esses dados podem ser observados nas Tabelas 1 e 2.

Os resultados permitiram identificar que, com relação à competência de desenvolvedor de programa, no domínio compreensão do planejamento do processo (denominado por I na Figura 1), a maior habilidade e também o maior desejo esteve em descrever e executar os passos básicos (estabelecer clima, avaliar necessidades, formular objetivos do programa, desenho e execução do programa e avaliação) do planejamento na educação de adultos (média $A^{1}=2,79$ e média $\left.\mathrm{D}^{2}=4,58\right)$, e a menor habilidade e menor desejo foram para usar estratégias de análise de sistemas em planejamento de programas (média $\mathrm{A}=2,37$ e média $\mathrm{D}=4,43$ ).

1 A - atual

2 D - desejado 
Tabela 1 - Docentes enfermeiros segundo variáveis sociodemográficas, tempo de atuação, carga horária e tipo de disciplina que ministra. São Paulo, Brasil. 2011.

\begin{tabular}{lcc}
\hline Variável & Frequência (total $=226)$ & Percentual (\%) \\
\hline $\begin{array}{l}\text { Faixa etária } \\
\quad 41 \text { a } 55 \text { anos }\end{array}$ & 119 & 52,65 \\
Sexo & 198 & 87,61 \\
$\quad$ Feminino & & 69,91 \\
Carga horária e tempo de docência & 158 & 77,88 \\
$\quad 31$ a 40 horas/ 6 a 10 anos & & 176 \\
$\quad$ Tipo de disciplina & & 76 rica e prática
\end{tabular}

Tabela 2 - Docentes enfermeiros segundo formação e educação permanente. São Paulo, Brasil. 2011.

\begin{tabular}{lcc}
\hline Variável & Frequência (total $=226)$ & Percentual $(\%)$ \\
\hline Formação & 122 & 53,98 \\
Escola pública & 101 & 44,69 \\
Escola privada & 3 & 1,33 \\
Não informado & 201 & 88,94 \\
PG Latu-senso & & 79,65 \\
PG Strictu-senso & 180 & 34,07 \\
Mestrado & 77 & 1,33 \\
Doutorado & 3 & 12,39 \\
Livre-docência & & 28 \\
Outras graduações & & \\
Sim & & \\
\hline
\end{tabular}

Com relação ao domínio planejamento e aplicação de programas (denominado por II na Figura 1), a menor habilidade esteve em construir desenhos de programas para atender às necessidades de diversas situações (treinamento de habilidades básicas, desenvolvimento de supervisores, gerentes e desenvolvimento organizacional) (média $A=2,43$ ), e a maior habilidade em desenvolver e executar plano de avaliação de programas de ensino (média $\mathrm{A}=$ $2,68)$. O maior desejo esteve em desenhar programas com variedade criativa de formatos, atividades, sequenciais, recursos e métodos avaliativos (média $\mathrm{D}=4,62$ ), e o menor desejo esteve em usar mecanismos de planejamento tais como conselhos, comitês e forças-tarefa (média $D=4,44$ ).

A relação entre as questões de cada domínio permitiu identificar, pela relação entre média e mediana de cada um, que os docentes estavam aquém do que desejavam alcançar (índice de significância 0,000), concentrando as respostas, na escala, em três para atual e cinco para desejado.
Figura 1 - Resultados sobre a competência Desenvolvedor de programas, relação entre domínios, nível atual e desejado para educação de adultos, segundo os docentes enfermeiros que atuam na graduação em enfermagem no município de São Paulo. 2011.

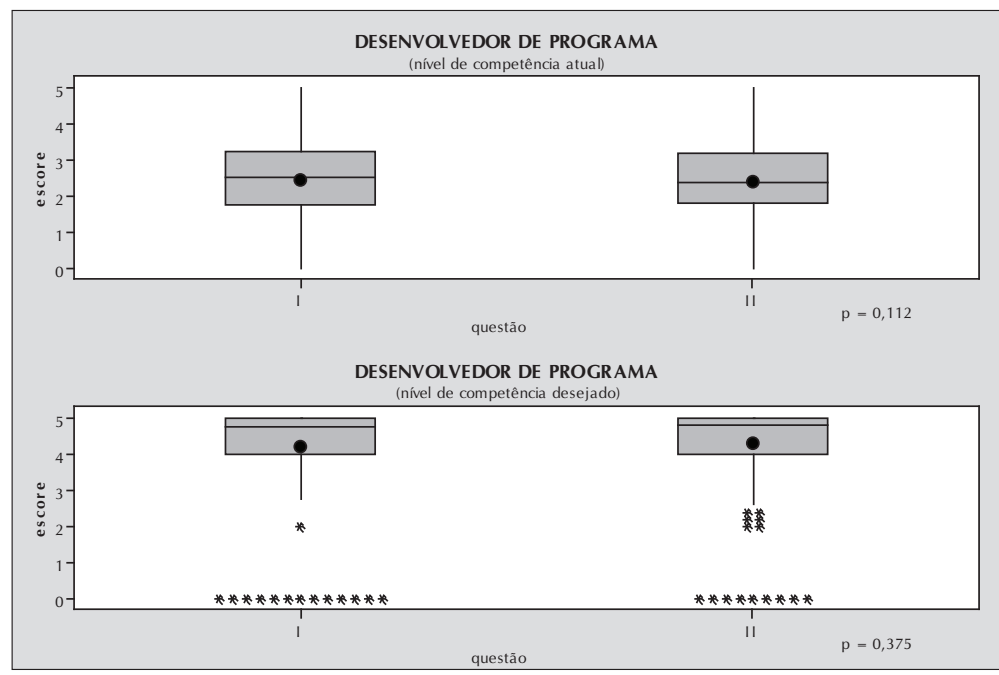


A Figura 1 indica que, na comparação entre os domínios das competências para desenvolver programas, os índices de significância não demonstraram relevância $(P(A)=0,112$ e $P(D)=0,375)$.

\section{DISCUSSÃO}

Trata-se predominantemente de uma população feminina, de meia idade, com pós-graduação, que atua em regime de aproximadamente 40 horas semanais e que ministra disciplinas teóricas e práticas. A Enfermagem apresenta predominância de mulheres e era esperado que os professores dessa área fossem desse sexo. $\mathrm{O}$ fato de boa parte ser de meia idade pode relacionar-se à necessidade da docência de nível superior requerer experiência profissional prática prévia e exigência de pós-graduação para ingresso nessa carreira. Terem se formado em escola pública pode estar relacionado a oportunidades de desenvolvimento vinculadas à escola pública onde o docente cursou graduação que, geralmente, tem tradição em pesquisa e pós-graduação. A quantidade de horas semanais corresponde à jornada de trabalho da maioria dos trabalhadores brasileiros e atende aos requisitos legais.

Os resultados dos dados sobre a titulação dos sujeitos dessa pesquisa apresentaram compatibilidade com a prescrição da Lei das Diretrizes e Bases da Educação Nacional no 9394/96 e o Decreto $\mathrm{n}^{\circ} 2.207 / 97^{(14)}$. Isso posto, entende-se que os professores são legalmente qualificados para o exercício da docência, pois atendem aos requisitos exigidos de formação. Nesse contexto, o que qualifica o professor para o exercício da docência é a pós-graduação estrito senso e, nesse aspecto, o mundo acadêmico revela o valor maior que dá às atividades de pesquisa, em comparação com as atividades de ensino.

Os saberes da docência são distintos dos da pesquisa, e os primeiros são menos valorizados na cultura universitária. Assim, o professor segue modelos profissionais de docentes de sua história pessoal, isto é, tem como base as experiências que teve ao longo de sua formação estudantil. Essa condição desfavorece inovações, pois são raros os professores que vivenciaram, como alunos, experiências inovadoras ${ }^{(15)}$.

Para as competências para desenvolver programas de educação de adultos, que serão discutidas a seguir, para todos os domínios, a maior parte dos professores declarou desempenho moderado e desejo máximo de desenvolver-se.

\section{A. Competência para desenvolver programas de educação de adultos}

\section{Compreensão do planejamento do processo}

Esse domínio reúne quatro habilidades. A maioria dos professores declarou habilidade atual moderada e desejo máximo nas tarefas sobre descrever e executar o processo andragógico (preparar o aluno, estabelecer clima, avaliar as necessidades, formular objetivos, desenho e execução do programa e avaliação), envolver representantes dos alunos no planejamento de programas e desenvolver e usar instrumentos e procedimentos de avaliação das necessidades de indivíduos, organizações e sociedade. Entretanto, os professores assinalaram deter menor habilidade para usar estratégias para analisar sistemas envolvendo planejamento de programas educativos. Análise de sistemas é uma ferramenta utilizada para desenvolver programas que envolve o desenho de fluxos, esquemas e diagramas por vezes altamente complexos e que incluem cálculos matemáticos avançados. Grande parte dos professores não desenvolve atividades de elaboração de projetos educativos, não detendo habilidades mais complexas nesse campo.

Por outro lado, construir, efetivar e avaliar projetos é prática rotineira na atividade docente universitária, que tem, na pesquisa, uma de suas funções predominantes. Para "projetar", enfermeiros são preparados nos programas de pós-graduação senso estrito, e isso é parte essencial da caminhada acadêmica(16).

Em algumas instituições, os professores são envolvidos em projetos de disciplinas, porém grande parte não colabora com a construção de projetos políticos pedagógicos, ficando isso sob a responsabilidade de grupos restritos de professores e coordenadores de cursos. Talvez por esse motivo, os docentes declararam não dominar com intensidade esse agrupamento de habilidades.

Vale ressaltar que professores devem dominar desenhos de programas de ensino para adultos que especifiquem o quê, onde e quando ensinar, bem como a sequência lógica do processo e o conteúdo que deve ser baseado em competências básicas, valores e ética, e voltados para a prática ${ }^{(17)}$.

Nesse rol de habilidades também se destaca a importância em se considerar, no desenvolvimento de programas, as características do aluno e de grupos heterogêneos que compõem as salas de aula que, por vezes, comportam indivíduos advindos de culturas diversas. Para populações heterogêneas ou culturalmente diferentes, como é o caso da população de alunos com quem os docentes participantes do presente estudo desenvolvem o processo de ensino-aprendizagem, há que se ajustar o programa à cultura dominante e estabelecer suporte social para que o aluno se adapte ao programa e o programa a ele. Isso significa humanizar o ensino. A Andragogia tem aproximação com a humanização por considerar a heterogeneidade dos alunos, privilegiando as experiências anteriores e a aplicação imediata do que se aprende, de modo a transformar a realidade do aprendiz ${ }^{(18)}$.

\section{Planejamento e aplicação de programas}

Esse domínio reúne cinco habilidades. A maioria dos professores declarou domínio atual moderado e desejo máximo, nas tarefas para desenvolver desenhos de programas que atendessem a diversas situações (treinamentos de habilidades básicas, desenvolvimento de professores, supervisores e organizacional), com variedade criativa de atividades, formatos, recursos e métodos, habilidade para usar conselhos, comitês e força-tarefa, e para avaliar o programa e atender aos requisitos institucionais.

O desenho de programas de ensino deve considerar os objetivos dos alunos, da instituição e da sociedade. A Andragogia comporta características de adultos que favorecem o estímulo e o desejo de aprender, sob a ótica da autodireção, em que o aluno é sujeito de seu aprendizado, que se motiva e se desenvolve a partir da concretização de suas conquistas. 
O aluno deve ser estimulado a atender à demanda social, e a instituição, por sua vez, deve transformar-se continuamente, para fazer a ponte entre o aluno e a sociedade ${ }^{(1)}$.

Processos andragógicos envolvem desenhos de programas ecléticos, criativos e variados, que estimulem a capacidade do aluno para se adaptar às mudanças, gerar novos conhecimentos e melhorar continuamente seu desempenho. Isso irá se refletir em práticas focadas em processos, com metas flexíveis e não prescritivas ${ }^{(19)}$.

Sob a ótica andragógica, professores devem facilitar a aprendizagem de modo que o aluno alcance resultados e competências. A facilitação está diretamente relacionada aos métodos escolhidos. Nesse contexto, alunos são responsáveis por selecionar métodos que facilitem sua aprendizagem e, para atender a esse requisito, estes devem assumir papel ativo em sua aprendizagem. Uma das dificuldades de programas educativos é mensurar a performance e níveis de competências adquiridos, e uma das maneiras de fazer isso é considerar os conhecimentos, as habilidades técnicas, objetivos alcançados e habilidades para o gerenciamento do tempo. Programas educativos com base na aquisição de competências facilitam a autoavaliação da performance do aluno, pois aproximam a teoria da prática e estimulam o processo de reflexão, que impulsiona o desejo de estar constantemente aprendendo para conquistar maior autonomia e novas competências ${ }^{(20)}$.

\section{B. Comparação entre as competências dos domínios para desenvolver programas de educação de adultos}

Para o desenvolvimento de programas educativos, a menor habilidade esteve em construir desenhos de programas para atender às necessidades de diversas situações (treinamento de habilidades básicas, desenvolvimento de supervisores e gerentes) e a maior habilidade em desenvolver e executar plano de avaliação de programas de ensino. O maior desejo esteve em desenhar programas com variedade criativa de formatos, atividades sequenciais, recursos e métodos avaliativos, e o menor desejo foi usar mecanismos de planejamento tais como conselhos, comitês e força-tarefa. Com isso, pode-se concluir que a maior parte dos docentes sabe executar suas ações educativas de maneira pedagógica tradicional e que transmitir a condução do aprendizado para o aluno ainda é algo pouco explorado e pouco experenciado. Consultar comitês de alunos implica em reconhecer essa autonomia, o que grande parte dos docentes ainda não está pronta para fazer, justamente porque a pedagogia tradicional não prescreve essas ações.

A comparação entre os domínios desse rol não foi significante estatisticamente, nem para o estado atual nem para o desejado, mostrando que os professores não viram diferença entre um domínio e outro, e que experenciaram essas competências menos que as de facilitação da aprendizagem, embora tenham declarado possuir habilidade moderada para as tarefas. Os docentes devem ser treinados para desenvolver esse papel. Geralmente essa tarefa fica a cargo de professores administradores que desenvolvem equipes e essa tarefa ainda não é comum, na maior parte das escolas de formação de enfermeiros.
As iniciativas dos órgãos reguladores do ensino superior para avaliação institucional têm a proposta de envolver os professores no desenvolvimento e administração de programas, mas, ainda assim, o distanciamento dessas tarefas é significativo e facilmente observável, nos resultados do presente estudo. O Sistema Nacional de Avaliação da Educação Superior (Sinaes) propõe a avaliação sob uma perspectiva formativa e com suporte para a formação de professores ${ }^{(9)}$. O Sinaes tem, por objetivo, analisar as instituições, os cursos e o desempenho dos estudantes. O processo de avaliação pretende levar em consideração aspectos como ensino, pesquisa, extensão, responsabilidade social, gestão da instituição e corpo docente. O Sinaes reúne informações do Exame Nacional de Desempenho de Estudantes (Enade), das avaliações institucionais e dos cursos. As informações obtidas devem ser utilizadas para orientação institucional de estabelecimentos de ensino superior e para embasar políticas públicas. Os dados também podem ser úteis para a sociedade, especialmente aos estudantes, como referência quanto às condições de cursos e instituições ${ }^{(11)}$. Na realidade, embora a avaliação de programas educativos seja do cotidiano das escolas, a proposta do sistema de avaliação Sinaes ainda é um devir, pois não atende, em sua plenitude, ao que propõe. Assim, fica explicado o perfil de respostas dos docentes pesquisados para esse conjunto de competências.

\section{CONCLUSÕES}

O presente estudo avaliou as competências para desenvolver programas educativos para adultos, dos docentes que atuavam em cusos de graduação de enfermagem paulistanos, por meio da Escala de Classificação Autodiagnóstica de Competências Andragógicas para o Papel do Educador de Adultos, desenvolvida por Malcom Shepherd Knowles ${ }^{(4)}$.

O instrumento demonstrou que é capaz de avaliar o perfil dos professores com relação às competências para atuar na educação de adultos e pode ser empregado em outros estudos. É desejavel a realização de outros estudos que pretendam replicar essa pesquisa em outras realidades, pois podem dar um panorama ampliado desta característica, o que poderia contribuir consideravelmente para os cursos de formação de professores, visto que há poucos referenciais nessa área, que é carente e necessita de avanços.

Os sujeitos do estudo eram predominantemente mulheres maduras, formadas em escolas públicas, mestres, com jornada de trabalho de 40 horas semanais, que atuavam entre 6 a 10 anos na docência, em disciplinas teóricas e práticas.

Com relação às competências para desenvolver programas educativos para adultos, os docentes declararam tê-las mais para compreender o planejamento de programas de ensino do que planejá-los e aplicá-los. Sobre o desejo de desenvolver-se nesse rol de competências para desenvolver programas, os sujeitos declararam interesse contrário, ou seja, desejar mais planejar e aplicar programas educativos do que compreender o planejamento de programas educativos.

Em resumo, o grupo de docentes sinalizou, de maneira geral, estar em nível intermediário em comparação ao máximo 
que desejava atingir dos agrupamentos de habilidades de facilitador de aprendizagem, ou seja, grande parte dos docentes enfermeiros que atuavam na graduação em Enfermagem no município de São Paulo em 2010 encontrava-se distante do ideal de nível máximo de competência que almejava. Assim, como esse grupamento valoriza e influencia consideravelmente o sucesso da aprendizagem na Enfermagem, sugere-se a intervenção nos processos de formação e aperfeiçoamento do professor de enfermagem, nessas competências, para a meIhoria do ensino.

A análise global dos resultados indicou ainda que, antes que qualquer intervenção que considere a Andragogia como fundamento para a formação de professores seja implementada, há que se mensurar o nível atual de competências desses indivíduos. Isso foi feito na presente pesquisa justamente porque não há, na Enfermagem, mensurações de competências na perspectiva andragógica proposta por Malcom Shepherd Knowles.

Os limites desse estudo devem ser considerados pelos leitores e pesquisadores, visto que se trata se um trabalho realizado em escolas e agrupamentos de professores característicos da cidade de São Paulo e que não cabem em outras realidades diferentes desta.

Considera-se, no entanto, que o estudo será útil para facilitar o planejamento de programas para desenvolvimento individual e institucional de professores e de instituições de ensino estudadas, e para os administradores, com relação às competências relevantes para aumentar a performance de docentes e atender melhor os alunos.

\section{REFERÊNCIAS}

1. Knowles MS, Holton III, Swanson R. Aprendizagem de resultados: uma abordagem para aumentar a efetividade da educação corporativa. Rio de Janeiro: Elsevier; 2009.

2. Knowles MS. Self-directed learning: a guide for learners and teachers. New York: Cambridge Adult Education, Globe Fearon; 1975.

3. Knowles MS. The making of an adult educator: an autobiographical journey. San Francisco: Jossey-Bassey; 1989.

4. Henry GW. An History Analisys of the development of thinking in the principal writings of Malcom Knowles School of Learning and professional studios. Queensland University of Technology. 2009.

5. Machado JLM, Caldas Júnior AL, Bortoncello NMF. Uma nova iniciativa na formação dos profissionais de saúde. Interface Comun Saúde e Educ 1997;1(1):147-56.

6. Draganov PB, Friedlander MR, Sanna MC. Andragogia na saúde: estudo bibliométrico. Esc Anna Nery Rev Enferm 2011;15(1):149-56.

7. Draganov PB, Carvalho AA, Sanna MC, Neves VR. Andragogia na enfermagem: uma revisão da literatura. Campo Grande: Associação Brasileira de Enfermagem. Anais do $16^{\circ}$ Seminário de Pesquisa de Enfermagem [evento na internet]. 2011 jun 19-22; Campo Grande, Brasil [acesso em 15 set 2011]. Disponível em: http://www.abeneventos.com.br/16senpe/senpe-trabalhos/files/0530.pdf

8. Knowles MS. The modern practice of adult education. New York: Association Press, \& Cambridge Book Publishers; 1980.

9. Ministério da Educação [homepage na internet]. Sistema Nacional de Avaliação da Educação Superior (Sinaes) [acesso em 15 maio 2011]. Disponível em: http://portal. inep.gov.br/superior-sinaes.

10. Ministério da Educação [homepage na internet]. Exame Nacional de Desempenho de Estudantes (Enade). [acesso em 15 maio 2011]. Disponível em: http://portal.inep.gov. br/enade.
11. Ministério da Educação (Brasil). Resolução CNE/CES, $\mathrm{n}^{\circ} .3$, de 7 de novembro de 2001.Institui diretrizes curriculares nacionais do curso de graduação em enfermagem. Diário Oficial da União 09 nov 2011 ;Seção 1.

12. Teixeira A. Educação e mundo moderno. São Paulo: Nacional; 1969.

13. Draganov PB. Avaliação das Competências Andragógicas dos Enfermeiros Docentes de Cursos de Graduação em Enfermagem do Município de São Paulo.São Paulo. Dissertação [Mestrado em Enfermagem] - Escola de Enfermagem da USP; 2011.

14. Organização das Nações Unidas para a Educação, a Ciência e a Cultura (UNESCO). Sexta Conferência Internacional de Educação de Adultos. CONFINTEA VI. Convergence [periódico na internet]. 2009 [acesso em 15 maio 2011];11(3-4). Disponível em: http://unesdoc.unesco.org/ images/0018/001861/186173por.pdf

15. Madison College [homepage na internet]. Adult learning theories in process education. [acesso em 15 maio 2011]. Disponível em: http://cetl.matcmadison.edu/efgb/2/2_1_2.htm

16. Anastasiou LGC. A construção de projetos como possibilidade educativa. Revista Educação PUC-Campinas 2006;(21):125-32.

17. Thompson JE. Competencies for midwifery teachers. Midwifery 2002;18(4):256-9.

18. Yurkovich EE. Working with american indians towards educational success. J Nurs Educ 2001;40(6): 259-69.

19. Henriksen E, Selander G, Rosenqvist U. Can we bridge the gap between goals and practice through a common vision? A study of politicians and managers' understanding of the provisions of elderly care services. Health Policy 2003;65(2):129-37.

20. Koerich MS, Erdmann AL. Enfermagem e patologia geral: resgate e reconstrução de conhecimentos para uma prática interdisciplinar. Texto \& Contexto Enferm 2003;12(4):528-37. 